\title{
Pathologic Evaluation of Skin Tumors With Ex Vivo Dermoscopy With Derm Dotting
}

Marc Haspeslagh, MD; Isabelle Hoorens, MD; Nele Degryse, BA; Ine De Wispelaere, BA; Annemarie Degroote, MD; Sarah Van Belle, BA; Jan Verboven, IR; Katrien Vossaert, MD; Fabio Facchetti, MD, PhD; Jo Van Dorpe, MD, PhD; Sofie De Schepper, MD, PhD; Lieve Brochez, MD, PhD

IMPORTANCE Ex vivo dermoscopy (EVD) with derm dotting (DD) improves clinicopathologic correlation and the quality of diagnosis in skin tumors.

OBJECTIVE To compare the diagnostic performance of the standard method of skin biopsy processing with the practice of EVD with DD.

DESIGN, SETTING, AND PARTICIPANTS This retrospective study compares the diagnostic performance in 6526 skin biopsy specimens examined from 2008 to 2010 with a standard method of processing with 8584 biopsy specimens examined in 2015 with EVD and DD. Data were analyzed from January 1 to March 31, 2016. A total of 15110 skin biopsy specimens were included. The biopsy specimens from 2008 to 2010 were processed in a hospital-based general pathology laboratory; the biopsy specimens from 2015 were processed in a private dermatopathology laboratory. Biopsy specimens from both periods were diagnosed by the same dermatopathologist.

MAIN OUTCOMES AND MEASURES The primary outcome measures were clinicopathological characteristics, usefulness of EVD with DD, and turnaround times (TATs).

RESULTS Use of EVD with DD increased the detection of positive section margins in nonmelanoma skin cancer from $8.4 \%$ to $12.8 \%$. The most significant increase was seen in Bowen disease, invasive squamous cell carcinoma, and a superficial type of basal cell carcinoma (BCC). With EVD and DD, a specific clinicopathologic diagnosis was made in $27.7 \%$ of nevi compared with only $10.3 \%$ using the standard method. The incidence of moderately and severely dysplastic nevi increased from $1.0 \%$ to $7.2 \%$ and from $0.6 \%$ to $1.4 \%$, respectively. The detection of ulceration in melanomas with thicker than $1 \mathrm{~mm}$ increased from $24.0 \%$ to $31.3 \%$. The number of nevi-associated melanomas increased from $15.5 \%$ to $33.3 \%$. The number of collision lesions from $0.07 \%$ to $1.07 \%$. The TAT for nevi decreased from 2 days to 1 day, for melanomas from 5 days to 2 days, and for BCC from 2 days to 1 day.

CONCLUSIONS AND RELEVANCE Ex vivo dermoscopy and DD with adapted sectioning in a dermatopathology setting allows a more accurate and less time consuming histopathologic diagnosis of skin tumors. These findings suggest that pathologists involved in skin tumor evaluation should be encouraged to learn dermoscopy and replace random transverse cutting with lesion-specific and DD-guided cutting.

JAMA Dermatol. 2017;153(2):154-160. doi:10.1001/jamadermatol.2016.4444 Published online December 28, 2016.
Supplemental content
Author Affiliations: Dermpat, Ghent, Belgium (Haspeslagh, Degryse, De Wispelaere, Degroote, Van Belle, Verboven); Department of Dermatology, Ghent University Hospital, Belgium (Haspeslagh, Hoorens, De Schepper, Brochez); private dermatology practice, Maldegem, Belgium (Vossaert); Department of Pathology, Spedali Civili, Brescia, Italy (Facchetti); Department of Pathology, Ghent University Hospital, Belgium (Van Dorpe).

Corresponding Author: Marc Haspeslagh, MD, Koning Albertlaan 210, 9000 Ghent, Belgium (info@dermpat.be). 
$\mathrm{M}$ ost pathology laboratories process skin biopsy specimens without access to clinical and/or dermoscopic images. Macroscopy of skin tumors is usually limited to a quick visual inspection, measurement of the lesion, standard transverse sectioning, and random cutting at 2 to 3 different levels.

Since the introduction of dermoscopy in the follow-up of pigmented skin lesions, focal areas that are visible only with dermoscopy may be the reason for diagnostic excision of lesions. Communication of this information to the pathologist may be of importance for a correct evaluation of these lesions. ${ }^{1}$ A routine diagnostic procedure submits only $2 \%$ or less of the excised tissue for examination. ${ }^{2}$ This is a worrisome fact, especially for melanocytic tumors, as one can imagine that small specific areas of concern for the clinician can be missed by the pathologist if these are not discernable by the naked eye. Merkel et al $^{3}$ applied a micropunch during in vivo dermoscopy to mark these areas of interest, which can, however, create an artifact and result in detaching of tissue.

In 2007, Scope et $\mathrm{al}^{4}$ described the added value that ex vivo dermoscopy (EVD) can give to the pathologist by guiding tissue sectioning. Cabete et $\mathrm{al}^{5}$ found that the combined use of EVD and histopathology improves clinicopathological correlation and allows selection of representative areas for sectioning. In $13.1 \%$ of their cases, a different final diagnosis was rendered after dermoscopy-guided specimen sectioning. Since 2011 we have systematically used EVD with derm dotting (DD) in our dermatopathology laboratory. With this simple and easy method, focal areas or suspected section planes observed on EVD are marked with nail varnish. This method of derm dotting was described by our team in $2013 .{ }^{6}$ Others have afterward implemented this method in the examination of pigmented lesions. ${ }^{7}$ The dotting method was also successfully used during oral surgery. ${ }^{8}$ In a systematic comparison of 101 tumoral skin lesions, we demonstrated that EVD is broadly similar to in vivo dermoscopy but also has clear differences, providing possible direction to target tissue for processing and examination. ${ }^{9}$

In the present study we evaluated the impact of EVD with DD on the reliability of pathological margin evaluation and accuracy of diagnosis. We also looked at the impact on time of this method by comparing additional cuts and turnaround time (TAT) (time from tissue cutting to pathological protocol).

\section{Methods}

Skin Biopsy Specimens and Histological Processing We compared 6526 skin biopsy specimens processed from January 1, 2008, to December 31, 2010, in a general pathology laboratory with 8584 skin biopsy specimens examined in a dermatopathology setting from January 1 to December 31, 2015. The skin biopsy specimens processed in this new dermatopathology setting can be considered comparable with those in the general hospital in terms of referred cases and patient population, since all the dermatologists sending to the general hospital pathology laboratory switched to the new dermatopathology laboratory. The number of skin biopsy speci-

\section{Key Points}

Question What is the diagnostic performance of processing skin tumors with ex vivo dermoscopy (EVD) and derm dotting (DD) compared with the standard method?

Findings We observed that EVD and DD significantly increase the detection of positive section margins in nonmelanoma cancers and result in a better recognition of specific clinicopathological subtypes of nevi; a higher incidence of moderately and severely dysplastic nevi, nevi-associated melanomas, and collision tumors; and a decrease in the median turnaround time for melanocytic tumors.

Meaning Ex vivo dermoscopy and DD permit a more accurate and less time-consuming histologic diagnosis of skin tumors.

mens increased through growth of the existing dermatology practices and by referral of cases from dermatologists at new private dermatology practices in the same region.

Because the submitting dermatology practices were all located outside the hospital, samples from both periods were considered comparable. The same dermatopathologist (M.H.) diagnosed the lesions from both periods.

The lesion specimens from the first time period were processed using the standard transverse method for skin biopsy specimen processing. In first instance, skin tumors were inspected by a technician by the naked eye. Measurements were taken, and a schematic drawing of lesion was made. The bottom side was inked. The lesion was standard bread-loaf cut every $3 \mathrm{~mm}$, with horizontal processing of tips. On 1 microscopic slide, 3 different levels of microtome cuts were assembled. Paraffin ribbons were conserved for possible additional staining.

Ex vivo dermoscopy and DD were used during the second time period. In the dermatopathology laboratory setting, skin tumors are inspected by a technician by the naked eye and measured. The technicians are trained in dermoscopy and decide if a dermoscopic image needs to be taken. A dermoscopic image (EVD, taken with a PowerShot G11, Canon; Dermlite 3, 3Gen Inc) was made of all flat melanocytic lesions, all lesions suspicious for dysplasia or skin cancer (both melanoma and nonmelanoma), and when specific dermoscopic information was provided by the clinician. Also, when a focal area of interest or suspect margin was noticed, a dermoscopic picture was made. When a technician was in doubt, the advice of the pathologist was asked. The images were only connected to the final histopathological diagnosis and no other personal data from patients were included. Moreover, according to Belgian law, no written informed consent was required. We therefore waived submission to the local human subjects ethics committee.

The following handling protocol was used (eFigure 1 in the Supplement): a horizontal border line was made on the skin rim with black nail varnish to orient the specimen (Figure 1). One half of the bottom side was inked green, the other half black. Focal lesions seen on dermoscopy were marked with a nail varnish dot. Different colors could be used. The varnish 
A Basal cell carcinoma in flat nevus

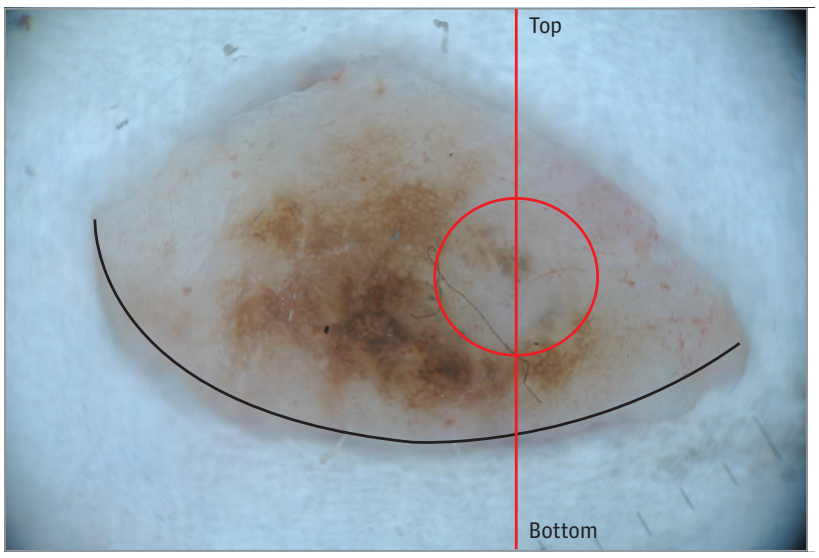

A, Dotting of the pale area in this pigmented lesion (circle) on the chest of a woman in her 40 s identified a superficial basal cell carcinoma in collision with a flat nevus. The vertical line indicates the section plane. A horizontal border line
B Original magnification $\times 12.5$

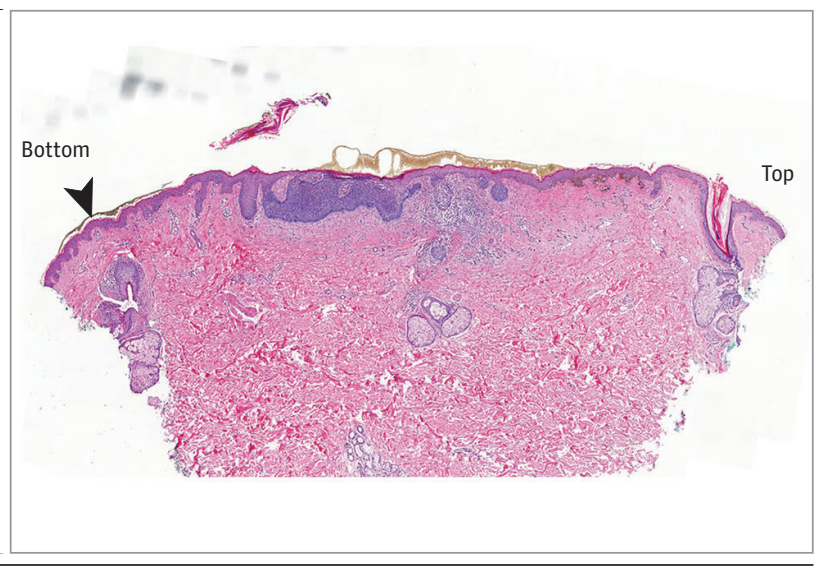

of black nail varnish was applied to orient the specimen (arrowhead). B, Histopathologic image, hematoxylin-eosin. A and B, "Top" and "bottom" indicate the top and bottom of the section plane. dot was discernable under the microscope as a granular browngray plaque on top of the horny layer (Figure 2). Punch biopsy specimens, taken to invest inflammatory diseases, were dotted in the center. Controlled microtome cutting was done until the area of interest (dotted) was reached. In addition, 2 previous depths were mounted on the same slide. Orientation, inking, nail varnish, and section planes were indicated on a drawing to help orient the tissue by the pathologist. Paraffin ribbons were conserved for possible additional staining.

Most benign melanocytic lesions were bread-loaf transversesectioned without processing of tips. In nonmelanoma skin cancer lesions, the tips were processed. In atypical melanocytic lesions and melanomas, the transverse sections were oriented to include the different structures, suspect margins, or focal lesions seen on EVD. In elongated lesions with clear transverse margins on EVD, horizontal or tangential sections were made. Acral nevi were cut perpendicular to the parallel ridge pattern.

\section{Outcome}

To evaluate the quality of the diagnostic process, the following features were systematically scored and compared for both periods: (1) the proportion of positive section margins in nonmelanoma skin cancer lesions; (2) the proportion of nevi subtypes; (3) the proportion of moderately and severely dysplastic nevi, melanoma in situ, and invasive melanomas; (4) the proportion of nevi-associated melanomas; (5) the incidence of ulceration in melanomas thicker than $1 \mathrm{~mm}$; and (6) the proportion of collision lesions.

To evaluate the economical and practical consequences of this extra macroscopic work, the number of deeper cuts and TATs from gross sectioning until the electronic validation of the definitive protocol was compared for both periods.

All categorical variables were compared using Pearson $\chi^{2}$ test. Median TAT was compared using Mann-Whitney test. All statistical tests were 2 -tailed, and $P<.05$ was considered sta- tistically significant. Statistical analysis was done using SPSS software (version 21.0; IBM).

\section{Results}

Positive Margins in Nonmelanoma Skin Cancer

With EVD and DD, the number of nonmelanoma skin cancer lesions with positive section margins increased significantly from $8.4 \%$ to $12.8 \%(P<.001)$ (Table 1$)$. In squamous cell carcinoma (SCC), the prevalence of positive margins increased from $8.7 \%$ to $16.9 \%$ ( $P$ < .001). For Bowen disease and in situ squamous carcinoma (ISCC), this number increased from $9.3 \%$ $19.1 \%(P=.02)$ and from $8.6 \%$ to $15.7 \%(P<.001)$, respectively. In the group of BCC, a positive margin was found in $11.1 \%$ compared with $8.3 \%$ without EVD and DD $(P=.02)$. In the superficial type of BCC, the incidence of positive margins increased from $1.8 \%$ to $4.8 \%(P=.046)$.

\section{Melanocytic Lesions}

Of 6526 biopsy specimens seen from 2008 to 2010, 1946 were diagnosed as nevi (29.8\%) compared with 2808 of 8584 (32.7\%) in 2015 (Table 2). For both periods, 2\% of biopsy specimens were melanomas. For Miescher, flat, blue, and spitzoid types of nevi, a similar incidence was found in the 2 periods (respectively, $15.6 \%$ vs $14.6 \%, 14.2 \%$ vs $14.8 \%, 2.5 \%$ vs $2.0 \%$, and $3.8 \%$ vs $3.2 \%)$. Only for Unna type nevi was a significant increase found (from $23 \%$ to $31.4 \%$ ). In addition to these common variants, with EVD and DD, 27.7\% of nevi were diagnosed as nevi with special clinicopathological characteristics compared with only $10.3 \%$ before using EVD and DD $(P<.001)$. Table 2 lists the incidence of these subtypes for both methods. The number of nevi with moderate dysplasia increased from $1.0 \%$ to $7.2 \%(P<.001)$, whereas the number of nevi with severe dysplasia increased from $0.6 \%$ to $1.4 \%(P=.005)$. 
In the periods 2008 to 2010 and 2015, similar numbers of melanoma in situ were observed (respectively, $0.6 \%$ and $0.7 \%$ ). Also, the incidence of invasive melanomas was similar for both periods $(1.3 \%$ and $1.4 \%)$. There was an increase in the diagnosis of residual nevi in melanomas from $15.2 \%$ to $33.3 \%(P=.06)$. A trend toward an increase was found for ulceration in melanomas thicker than $1 \mathrm{~mm}$ (from $24.0 \%$ to $31.3 \% ; P=.54$ ).

\section{Collision Lesions}

Ex vivo dermoscopy and DD revealed a collision lesion in $1.1 \%$ of the cases, compared with only $0.07 \%$ of cases diagnosed between 2008 and $2010(P<.001)$.

\section{Deeper Cuts}

Comparing both periods, a slight, nonsignificant, increase in demand for deeper cuts was found using EVD and DD (6.4\% vs $5.3 \%$ using the standard method). For nonmelanoma skin cancer, a decrease was found (from 3.8\% to 2.9\%). For nevi, deeper cuts were asked in 5.3\% of cases, compared with 5.1\% with the standard method. For melanomas, there was an increase from $25.5 \%$ to $57.9 \%$.

\section{Turnaround Time}

The median TAT significantly decreased during the second time period from 2 days to 1 day for nevi, from 5 to 2 days for melanoma, and from 2 to 1 day for BCC $(P<.001)$.

\section{Discussion}

The significant increase in positive section margins in nonmelanoma skin cancer lesions (from $8.4 \%$ to $12.8 \%$ ) indicates a higher sensitivity of the EVD and DD method. In ISCC, the visualization of a yellow-brown keratotic or crusty surface on EVD permits delineation of the lesion and can guide us to suspicious margins. Many Bowenoid lesions show skip lesions. They may be covered with a keratotic crust or show brown dots corresponding to residual glomerular vessels. ${ }^{9}$ These characteristics on EVD can disclose a positive margin and guide the tissue sectioning. In BCCs, EVD with DD proved to increase the number of positive margins significantly (from 8.3\% to $11.1 \%$ ). This was mainly due to superficial BCCs that may grow multifocal and may not be well delineated clinically.

As we described in a previous report, ${ }^{9}$ in some BCCs, the loss of red and intensification of the white structureless areas on EVD (reflecting a fibrosing stromal reaction) permits a better margin evaluation. ${ }^{6}$ In other cases of superficial BCCs, small brown crusts or blue-gray ovoid nests (corresponding to pigmented basal nests) may give a hint to the most marginal tumoral nests. Nodular BCCs are mostly well delineated clinically, and surgery often results in clean margins. Margin positivity in these cases are mostly found in the deeper section plane or deeper lateral sides, and this information cannot be captured by EVD.

As demonstrated in a previous study, ${ }^{9}$ EVD of pigmented lesions provides a sharp and detailed picture, allowing an excellent orientation and guided cutting of the lesions. Suspicious superficial borders can easily be identified (Figure 2).

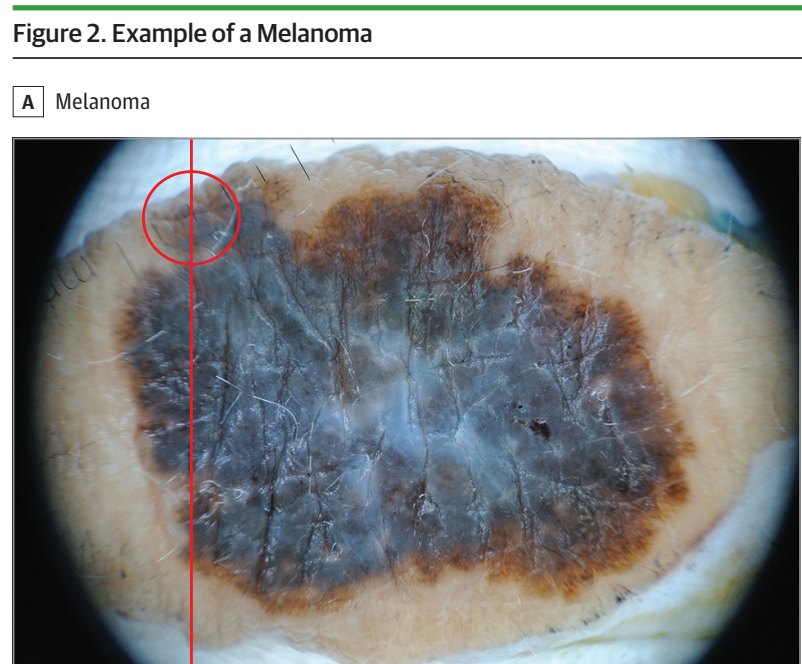

B Original magnification $\times 12.5$

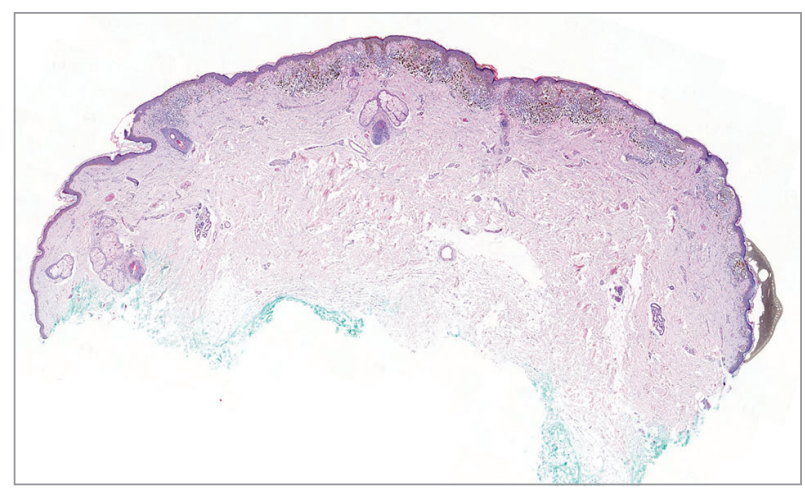

Original magnification $\times 50$

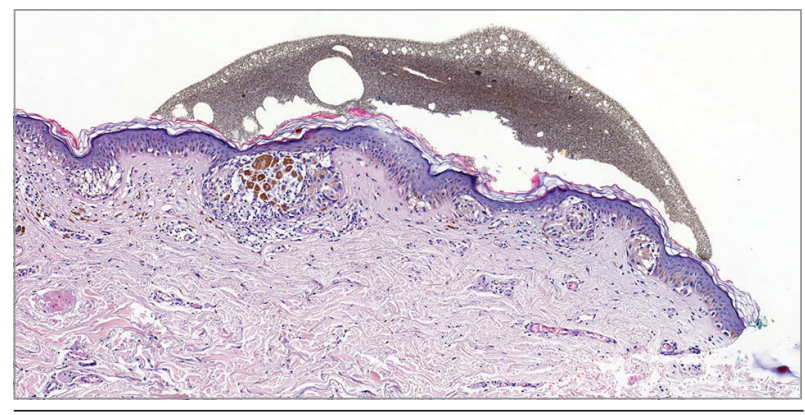

A, This superficial spreading melanoma with a thickness of $0.6 \mathrm{~mm}$ on the clavicula of a woman in her 60s shows on ex vivo dermoscopy a focal suspicious margin (circle), confirmed to be positive after dotting and guided cutting. The line indicates the section plane. B and C, Histopathologic images, hematoxylin-eosin.

During both investigated periods, a similar percentage of common variants of nevi was found. Only for Unna-type nevi was a significant increase in the amount of nevi found (from $23 \%$ to $31.4 \%$ ). This probably reflects more attention for subtyping and a lower threshold for diagnosing a nevus as papillomatous since the use of EVD.

In $27.7 \%$ of nevi, many of which were removed because of clinically atypical aspects, EVD with DD led to a more specific clinicopathologic subtyping (27.7\% vs $10.3 \%$ without EVD and 


\begin{tabular}{|c|c|c|c|}
\hline Type of Tumor & $\begin{array}{l}\text { General Hospital Period, } \\
2008-2010, \text { No./Total } \\
\text { No. (\%) }\end{array}$ & $\begin{array}{l}\text { Private Dermatopathology } \\
\text { Period, 2015, } \\
\text { No./Total No. (\%) }\end{array}$ & $P$ Value \\
\hline Nonmelanoma skin cancer & $130 / 1540(8.4)$ & $239 / 1874(12.8)$ & $<.001^{\mathrm{a}}$ \\
\hline Squamous cell carcinoma & $41 / 471(8.7)$ & $89 / 524(16.9)$ & $<.001^{\mathrm{a}}$ \\
\hline Bowen disease & $12 / 129(9.3)$ & $37 / 194(19.1)$ & $.02^{\mathrm{a}}$ \\
\hline In situ NOS & $1 / 10(10.0)$ & $3 / 9(33.4)$ & .21 \\
\hline Keratoacanthoma type SCC & $3 / 48(6.3)$ & $5 / 41(12.1)$ & .33 \\
\hline Invasive SCC, other & $25 / 284(8.6)$ & $44 / 280(15.7)$ & $<.001^{\mathrm{a}}$ \\
\hline Basal cell carcinoma & $89 / 1069(8.3)$ & $150 / 1350(11.1)$ & $.02^{\mathrm{a}}$ \\
\hline Superficial & $5 / 276(1.8)$ & $16 / 336(4.8)$ & $.046^{\mathrm{a}}$ \\
\hline Superficial/nodular & $3 / 11(27.3)$ & $12 / 103(11.7)$ & .15 \\
\hline Nodular/noduloulcerative/nodulocystic & $62 / 710(8.7)$ & $82 / 725(11.3)$ & .10 \\
\hline Nodular/infiltrative & $2 / 8(25.0)$ & 2/13 (15.4) & .59 \\
\hline Infiltrative/morpheiform & $4 / 8(50.0)$ & $28 / 102(27.5)$ & .18 \\
\hline Basosquamous & $4 / 21(19.0)$ & $1 / 6(16.7)$ & .89 \\
\hline Others or NOS & 9/63 (14.3) & 9/65 (13.8) & .94 \\
\hline
\end{tabular}

Abbreviations: NOS, not otherwise specified; SCC, squamous cell carcinoma.

a Statistically significant.

\begin{tabular}{|c|c|c|c|}
\hline Type of Nevus & $\begin{array}{l}\text { General Hospital Period, } \\
\text { 2008-2010, No. (\%) }\end{array}$ & $\begin{array}{l}\text { Private Dermatopathology Period, } \\
2015 \text {, No. (\%) }\end{array}$ & $P$ Value \\
\hline Total biopsies & 6526 & 8584 & \\
\hline Nevi & 1946 of $6526(29.8)$ & 2808 of $8584(32.7)$ & $<.001^{\mathrm{a}}$ \\
\hline Unna & $449(23.0)$ & $884(31.4)$ & $<.001^{\mathrm{a}}$ \\
\hline Miescher & $303(15.6)$ & $409(14.6)$ & .34 \\
\hline Flat & $277(14.2)$ & $416(14.8)$ & .58 \\
\hline Blue & $49(2.5)$ & $55(2.0)$ & .20 \\
\hline Spitz/Reed & $74(3.8)$ & $91(3.2)$ & .30 \\
\hline Other & $418(21.5)$ & $420(15.0)$ & $<.001^{\mathrm{a}}$ \\
\hline NOS & $376(19.3)$ & $533(19.0)$ & .77 \\
\hline Clinicopathologic subtypes & $200(10.3)$ & $777(27.7)$ & $<.001^{\mathrm{a}}$ \\
\hline Flat hyperpigmented/lentiginous & $158(8.1)$ & $236(8.4)$ & .21 \\
\hline Large nested & $23(1.2)$ & $63(2.2)$ & $<.001^{\mathrm{a}}$ \\
\hline Actively growing & 0 & $35(1.2)$ & $<.001^{\mathrm{a}}$ \\
\hline Asymmetric shoulder & 0 & $97(3.4)$ & $<.001^{a}$ \\
\hline Fibrosing/sclerosing & $6(0.3)$ & $168(6.0)$ & $<.001^{\mathrm{a}}$ \\
\hline Sclerosing & $6(0.3)$ & $104(3.7)$ & $<.001^{\mathrm{a}}$ \\
\hline Superficial fibrosing & 0 & $37(1.3)$ & $<.001^{\mathrm{a}}$ \\
\hline Perifollicular fibrosing & 0 & $27(1.0)$ & $<.001^{\mathrm{a}}$ \\
\hline Nevus with dot & 0 & $26(0.9)$ & $<.001^{a}$ \\
\hline Irritative/traumatized & $1(0.1)$ & $118(4.2)$ & $<.001^{\mathrm{a}}$ \\
\hline Eczematized & $5(0.3)$ & $17(0.6)$ & .08 \\
\hline Recurrent & $7(0.4)$ & $17(0.6)$ & .24 \\
\hline Nevi with moderate dysplasia & $20(1.0)$ & $201(7.2)$ & $<.001^{a}$ \\
\hline Nevi with severe dysplasia & $11(0.6)$ & $40(1.4)$ & $.008^{a}$ \\
\hline Melanoma in situ & $42(0.6)$ & $59(0.79)$ & .74 \\
\hline Invasive melanomas & $87(1.3)$ & $116(1.4)$ & .80 \\
\hline Ulceration in melanomas $>1 \mathrm{~mm}$ & 6 of $25(24.0)$ & 10 of $32(31.3)$ & .54 \\
\hline Nevi-associated melanomas & 5 of $33(15.2)$ & 18 of $54(33.3)$ & $.06^{\mathrm{a}}$ \\
\hline
\end{tabular}

Abbreviation: NOS, not otherwise specified.

a Statistically significant.
DD) (Table 2). The only nevus subtype that could be easily identified without EVD and DD were hypermelanotic flat and/or lentiginous nevi (8.4\% vs $8.1 \%$ in 2015). Diagnoses of large nested nevus increased from $1.2 \%$ to $2.2 \%$. Ex vivo dermoscopy and adapted cutting may have contributed to a better understanding of the specific architecture of melanocytic lesions. After correlation with EVD, $1.2 \%$ of nevi were diagnosed as actively growing nevi with peripheral globules, corresponding to another spe- 
Figure 3. Example of Actively Growing Nevus

\section{A Nevus}

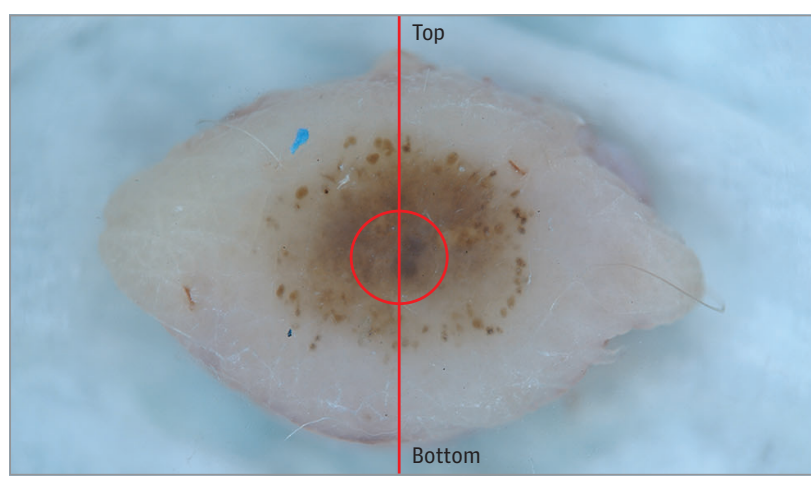

C Original magnification $\times 200$

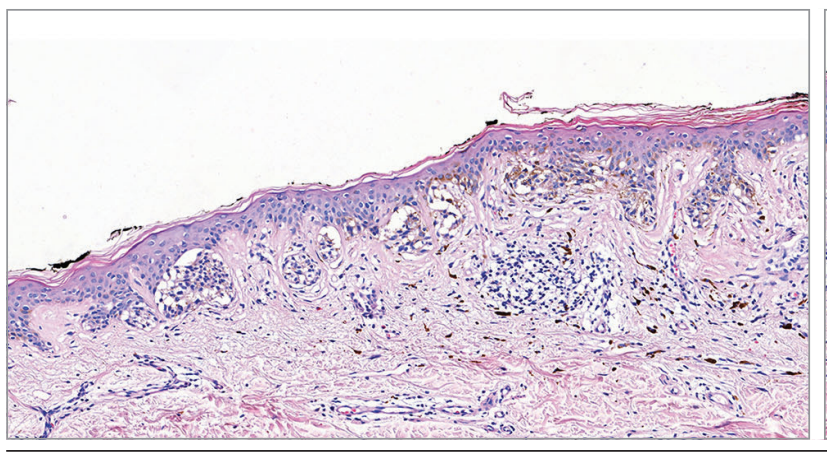

A This pigmented lesion on the chest of a man in his 20s with a history of growth in the past 6 months shows a homogeneous center and a separated peripheral rim of brown globules corresponding to a flat compound nevus. The dotted blue-gray center (circle) corresponds to an area of chronic inflammation
B Original magnification $\times 12.5$

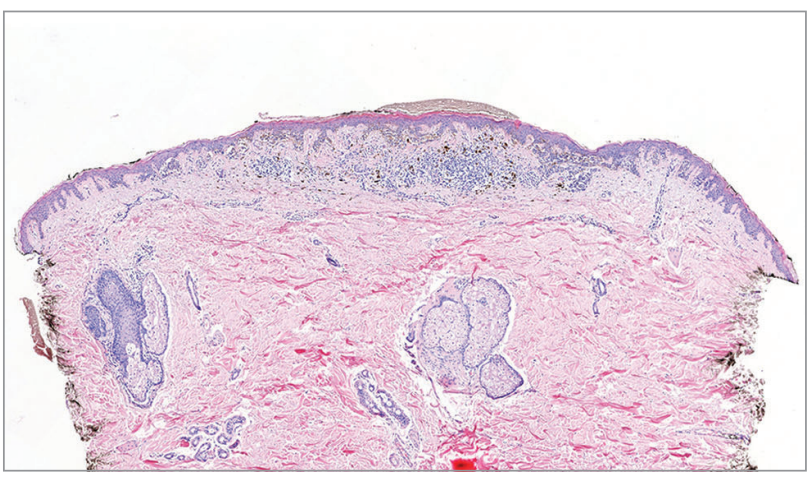

D Original magnification $\times 200$

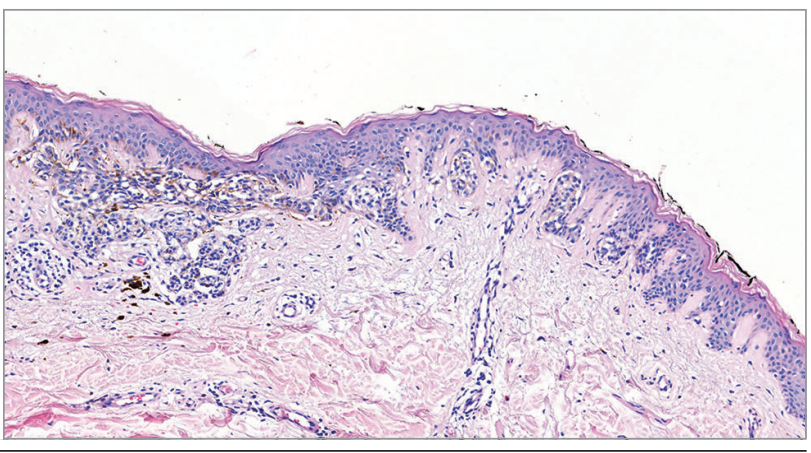

and melanophagia. The line indicates the section plane. B-D, Histopathologic images, hematoxylin-eosin. C and D, The peripheral rim of globules corresponds to larger or fused junctional nests. cific nevus with large nests (Figure 3). This diagnosis is almost impossible to make without dermoscopic information.

Focal areas of fibrosis and/or sclerosis in nevi may cause concern for the clinician and often prompt resection, but these areas may be difficult to trace without EVD and DD. The diagnosis of a fibrosing or sclerosing nevus by use of EVD was made in $6 \%$ of nevi compared with only in $0.3 \%$ without EVD. The latter were all examples of fully sclerosing nevi. Many fibrosing, sclerosing nevi however, show only focal sclerosis, often starting in a perifollicular way. Without EVD and DD these areas can be missed. A correct interpretation of fibrosing/ sclerosing nevi is important because the differential diagnosis of these cases with regressive melanoma can be very difficult, even with the aid of EVD and DD information.

Nevi that were resected as consequence of the appearance of a new black, blue, or brown dot were marked at this dot and diagnosed readily through EVD without need for deeper cuts or risk of transection of the dot.

Irritated, traumatized nevi were also significantly more frequently diagnosed than previously. There was also an increase in the diagnosis of recurrent and eczematized nevi. This increase, probably owing to the low number, was not significant. Ex vivo dermoscopy often revealed focal changes in these lesions (crusts, erosions, pigment changes), permitting marking and guided cutting.

Ex vivo dermoscopy with DD resulted in a significant increase of moderately and severely dysplastic nevi, from $1.0 \%$ to $7.2 \%$ and from $0.6 \%$ to $1.4 \%$, while the incidence of melanoma in situ, an invasive melanoma, remained the same. This observation suggests a higher sensitivity of EVD with DD specifically for the identification of areas of significant dysplasia. This is in line with the retrospective study of Merkel et $\mathrm{al}^{9}$ on the use of dermoscopy-guided histologic sectioning for the diagnosis of melanocytic lesions. This has clinical consequences because in severely dysplastic nevi a broader resection to $0.5 \mathrm{~cm}$ is advised.

Besides an adequate control of section planes, EVD with DD may also help to identify a preexisting nevus rest in melanomas or a focus of melanoma in a benign nevus. The observation of small areas with a residual regular network or conserved globules in a dermoscopically malignant lesion can disclose a preexisting nevus. Focal areas with a disturbed network or a blue-gray aspect on EVD can guide the pathologist to a small focus of melanoma in a benign nevus. By dotting and thereby facilitating microscopic traceability of these areas, there was an increase in the diagnosis of residual nevi in melanomas 
(from $15.2 \%$ to $33.3 \%$ ). The diagnosis of ulceration in melanomas thicker than $1 \mathrm{~mm}$ increased from $24.0 \%$ to $31.3 \%$. However, this increase was not significant, probably owing to the low number of cases included in this study. The diagnostic potential of EVD and DD in identifying ulceration in melanomas has to be further evaluated in larger series. Ex vivo dermoscopy and DD can also help to identify the most suspicious areas for deep invasion in melanomas. These areas often show a white-gray color as sign of regression or a blue-gray aspect owing to the presence of deeper dermal melanin pigment.

By using EVD and DD, we observed more collision lesions $(0.07 \%$ to $1.07 \%)$. This increase may indicate a higher sensitivity of the new method compared with the standard method. Although most of these collision lesions are incidental findings, without clinical or therapeutic consequences, in some of these cases, the identification of a second lesion can be of clinical importance (Figure 1).

Turnaround times decreased in the second period compared with the first period: for nevi (from 2 days to 1 day), melanomas (from 5 days to 2 days), and BCC (from 2 days to 1 day) significantly. In the private dermatopathology laboratory we process tissues twice a day compared with only 1 overnight run in the general pathology hospital laboratory, but this explains only in part the low TATs.

Deeper cuts were required in $6.4 \%$ of cases compared with $5.3 \%$ before, which is a nonsignificant difference. The need for deeper cuts is low compared with that reported in a previous study (7.6\% to $23.3 \%) .{ }^{10}$ With EVD-guided sectioning, most deeper cuts are made to confirm or further examine focal lesions observed on the first cuts, whereas with the standard method, cuts are often blind because of a discordance between the histological findings and the expected findings, based on the clinical information or macroscopy. The exact impact on the accuracy and efficiency of working in an exclusively dermatopathology environment compared with that in a general pathology laboratory is difficult to measure. Nevertheless, we are convinced that EVD and DD may contribute in improving the accuracy and efficiency in dermatopathology. The main limitation of the study is that for the second period the biopsies were processed in a new dermpat lab.

\section{Conclusions}

This retrospective evaluation shows that EVD with DD and adapted sectioning of skin tumors, permits a more accurate histologic diagnosis. It allows better margin evaluation, better understanding of lesional architecture, and specific evaluation of remarkable/suspicious areas, either described by the clinician or observed by the laboratory technician. The method is easy to implement in a dermatopathology setting. In our opinion, EVD with DD is a valuable new scientific tool to make clinicopathologic correlations. We believe it is time for the pathologist involved in tumoral skin evaluation and their technical staff to learn dermoscopy and replace random transverse cutting by lesion-specific and DD guided cutting.

\section{ARTICLE INFORMATION}

Accepted for Publication: September 26, 2016.

Published Online: December 28, 2016. doi:10.1001/jamadermatol.2016.4444

Author Contributions: Dr Haspeslagh had full access to all of the data in the study and takes responsibility for the integrity of the data and the accuracy of the data analysis.

Concept and design: Haspeslagh, Hoorens, Degryse, De Wispelaere, Vossaert, Facchetti, De Schepper، Brochez.

Acquisition, analysis, or interpretation of data: Haspeslagh, Hoorens, Degryse, Degroote, Van Belle, Verboven, Vossaert, Van Dorpe, Brochez. Drafting of the manuscript: Haspeslagh, Degryse, De Wispelaere, Verboven.

Critical revision of the manuscript for important intellectual content: Haspeslagh, Hoorens, Degryse, Degroote, Van Belle, Vossaert, Facchetti،

Van Dorpe, De Schepper, Brochez.

Statistical analysis: Haspeslagh, Hoorens, Van Belle. Administrative, technical, or material support: Haspeslagh, Degryse, De Wispelaere, Verboven, De Schepper.

Supervision: Van Dorpe.

Conflict of Interest Disclosures: None reported.

\section{REFERENCES}

1. Ferrara G, Annessi G, Argenyi Z, et al. Prior knowledge of the clinical picture does not introduce bias in the histopathologic diagnosis of melanocytic skin lesions [published online August 13, 2015]. J Cutan Pathol. doi:10.1111/cup.12589

2. Dyson SW, Bass J, Pomeranz J, Jaworsky C, Sigel J, Somach S. Impact of thorough block sampling in the histologic evaluation of melanomas. Arch Dermatol. 2005;141(6):734-736.

3. Merkel EA, Amin SM, Lee CY, et al. The utility of dermoscopy-guided histologic sectioning for the diagnosis of melanocytic lesions: a case-control study. J Am Acad Dermatol. 2016;74(6):1107-1113.

4. Scope A, Busam KJ, Malvehy J, et al. Ex vivo dermoscopy of melanocytic tumors: time for dermatopathologists to learn dermoscopy. Arch Dermatol. 2007;143(12):1548-1552.

5. Cabete J, Lencastre A, João A. Combined use of ex vivo dermoscopy and histopathology for the diagnosis of melanocytic tumors. Am J Dermatopathol. 2016;38(3):189-193.

6. Haspeslagh M, Degryse N, De Wispelaere I. Routine use of ex vivo dermoscopy with "derm dotting" in dermatopathology. Am J Dermatopathol. 2013;35(8):867-869.
7. Salkus G, Hansen MH, Erentaite D. Ex vivo dermoscopy with "derm dotting" in routine dermatopathology practice: our experience in 1 year. Dansk Patologiselskabs Årsmøde 2015; Nyborg, Denmark; Abstract 0-4-7. http://www .danskpatologi.dk/doc/aarsmoede/2015_Abstracts \%20endelig\%20opsaetning\%20(1).pdf. Accessed November 23, 2016

8. Van Hevele J, Hauben E, Haspeslagh M, et al. Application of derm dotting in oral and maxillofacial surgery. Oral Sci Int. 2016;13(1):20-23. doi:10.1016 /S1348-8643(15)00021

9. Haspeslagh M, Vossaert K, Lanssens S, et al. Comparison of ex vivo and in vivo dermoscopy in dermatopathologic evaluation of skin tumors. JAMA Dermatol. 2016;152(3):312-317.

10. Mudaliar K, Speiser J, Hutchens KA. Changing dermatopathology protocols for processing small skin biopsies: decreases in slide quantity does not affect patient care and allows for optimal efficiency. Am J Dermatopathol. 2016;38(6):409-412. 


\section{NOTABLE NOTES}

\section{Stanisław Ostrowski, MD-The One State President Among Dermatologists in the World}

Martyna Woltanowska, MD; Andrzej Wincewicz, MD, PhD, FEBP

Stanisław Ostrowski was born on October 29, 1892, in Lvov, Poland. He studied medicine in Lvov-with partial education in Vienna, Austria-and obtained his medical degree in 1919 in Lvov. When Poland struggled for independence, his other mission was military service. He belonged to Polish patriotic organizations, such as the Riflemen's Association and the Union of Active Struggle, and graduated from officer school and served as a soldier and medical physician in Joseph Pilsudski's First Brigade and Medical Chancellery of Legions during World War I. He defended Lvov in 1918 and in 1920 was the chief medical physician of the infantry regiment. In 1920, he was honored with the medal Orlęta ("Eaglets"), a commemorative order for the Lvov defenders against the Soviets.

Ostrowski was employed at the Department of Dermatology at Warsaw University from 1922 to 1925 . After he returned to Lvov, he became the chief of dermatology ward of State General Public Hospital in Lvov, and in 1931 he became an associate professor. ${ }^{1,2}$ He investigated the histology of unilateral epitheliomatous sebaceus nevus of the head. ${ }^{3}$ He was a brilliant clinician who published his meticulous observations about comparing 2 clinical appearances of tuberculids of infant skin; attempted to cure different manifestations of tuberculosis with a tuberculin cloth pad to enhance immunological response on affected skin surfaces; published modifications of blood morphology in rickets and comparative studies of the Mantoux tuberculin skin test; examined light-sensitizing properties of acridine derivatives in treatment of psoriasis and reported 20 years of experience with the efficacy of novarsenobenzol in treating lues; studied vegetative nervous reactions in tuberculosis and lues ${ }^{3}$; and described the dermatological adverse effects of various contemporary medications (eg, gold and arsenobenzole) as well as consequences of poisoning with venoms.
In 1930, Ostrowski was elected to the Polish parliament, and in 1934 was appointed vice president of the city of Lvov. He was elected president of this city and held that office from June 24,1936 , to September 23 , 1939. After the Soviet invasion of Eastern Poland on September 17, 1939 , he was imprisoned by the Soviets in Lvov and later transferred to Russia (Lubianka, Butyrki, Moscow). As part of the amnesty agreement of 1941, he left the work camp in Krasnoyarsk, Siberia. During World War II, he took part in battle campaigns in Italy with the army of General Wladyslaw Anders. After the war, he supervised the dermatology department in Penley, Wales, and later worked in the Military Hospital, Mossley Hill, Liverpool, England, until his retirement in 1962. He was the president of the Republic of Poland in exile from April 9, 1972, to April 8, 1979. He died in Charing Cross Hospital in London on November 22, 1982. ${ }^{1,2}$

Author Affiliations: Non-Public Health Care Unit "IL-MED," Department of Family Medicine, Szudziałowo, Poland (Woltanowska); Non-Public Health Care Unit, Department of Pathology (NZOZ Zakład Patologii Spółka z o.o.), Kielce, Poland (Wincewicz).

Corresponding Author: Andrzej Wincewicz MD, Non-Public Health Care Unit, Department of Pathology (NZOZ Zakład Patologii Spółka z o.o.), Kielce, ul. Jagiellońska 70, 25-734 Kielce, Poland (ruahpolin@yahoo.com).

Additional Contributions: We are indebted to everyone who cares about the commemoration of the eminent professors of King Jan Casimir University in Lvov.

1. Łomaczewska DB. Stanisław Ostrowski-Last President of Royal and Capital City of Lvov [in Polish]. http://www.Iwow.com. pl/Biuletyn/testament.html. Accessed January 26, 2016.

2. Stanisław Ostrowski. Wikipedia. https://en.wikipedia.org/wiki/Stanis\%C5 \%82aw_Ostrowski. Accessed January 26, 2016.

3. Data from Section of Special Collection, Stanisław Konopka Main Medical Library Online catalog of 1901-1939 publications. http://195.187.98.5/. Accessed July 18, 2014. 